\title{
Characterization of confined liquid jet injected into oscillating air crossflow
}

\author{
Virginel Bodoc, Anthony Desclaux, Pierre Gajan, Frank Simon, Geoffroy Illac
}

Received: date / Accepted: date

\begin{abstract}
The aim of this work is to study the role of the liquid phase in the thermo-acoustic coupling that leads to combustion instabilities. In multipoint injection systems, the liquid fuel is injected through orifices as jets which are destabilized by the high-speed air flowing in a transverse direction. This paper is focused on the effect of an acoustic excitation imposed on the air flow on the various liquid features observed in actual fuel injection systems. This concerns the initial jet trajectory, its breakup, the formation and transport of the resulting spray, the liquid film formation on walls and its final atomization at the outlet of the injection system. Experimental investigations were performed on a simplified geometry designed to reproduce these main phenomena. Phaseaveraged processing of the experimental data is used to analyze the relationship between the acoustic excitation and the liquid phase behavior. In particular, the phase delays imposed by the various processes involved are analyzed. The processing of high-speed video recordings reveals harmonic oscillation of the liquid column which periodically breaks into a spray structure or impacts the opposite wall, forming a liquid film. Using PDA measurements, it is shown that the resulting two-phase flow consists of alternating dense and diluted zones transported by the air flow. At the end of the channel, the liquid film flowing on the wall opposite the liquid jet is periodically atomized through the oscillating shear forces imposed by the air flow.
\end{abstract}

Keywords: thermo-acoustic instabilities, liquid jet, cross flow, acoustics

\section{Introduction}

Confined devices with an internal heat source are predisposed to thermo-acoustic instabilities, resulting from a coupling between the acoustic field, pressure and velocity fluctuations (respectively $\mathrm{p}^{\prime}$ and $\mathrm{u}^{\prime}$ ), and the unsteady heat release $\left(\mathrm{q}^{\prime}\right)$. This phenomenon was first observed by Higgins [1] and Rijke [2] and explained by Lord Rayleigh in 1878 [3]. During the second half of the 20th century, many studies first focused on instabilities occurring in liquid propellant rockets [4] and later on other combustion devices such as industrial furnaces, boilers, or other propulsion systems like solid rockets, gas turbines or afterburners. For these different applications, the coupling line between the acoustic components and the unsteady heat release greatly depends on the combustion regime (premixed or not), the reactant characteristics and state (solid, liquid or gaseous) and the injection procedure. 
Likewise, the feedback loop between the unsteady heat release and the acoustic state depends on the device geometry, its boundary conditions, and the Mach number distribution. As a result, each configuration induces specificities that must be taken into account (Gajan et al. $[5])$.

Over the last few decades, many studies on combustion instabilities have concerned landbased turbine or aero engines. In these fields (energy production or civil air transport), manufacturers strive to reduce the environmental impact by modifying their engine design. In particular, for two decades, they have been developing shorter combustion chambers operating at a higher Overall Pressure Ratio (OPR) in lean combustion regimes, in order to minimize pollutant formation (NOx, soot, etc.). In these new combustors, a higher air mass flow rate is required for the combustion; therefore less air is available for cooling the combustor walls. Consequently, walls with multiple perforations are generally used for cooling, which modifies their acoustic impedance. Some of these geometric modifications may enhance the onset of combustion instabilities, which lead to large cyclic pressure or velocity fluctuations inside the chamber and, consequently, generate significant heat transfer at the combustor walls, or large amplitude vibrations of the structure. This can result in damage to the combustor or even to its destruction, which is incompatible with the safety and durability standards required in the aeronautical industry.

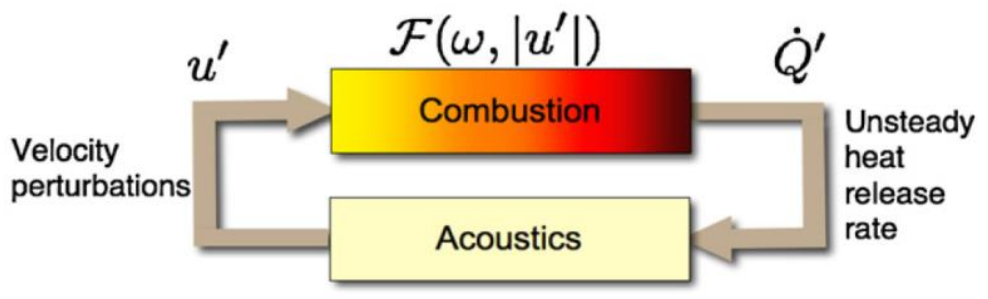

Fig. 1: Coupling loop at the origin of thermo-acoustic instabilities The flame response is conveniently represented in terms of a describing function $\mathrm{F}\left(\omega,\left|\mathrm{u}^{\prime}\right|\right)[6]$.

The p'q' coupling can be described as a closed loop of interactions, back and forth, between acoustic and heat release, as schematically represented in Fig. 2 [6]. It was shown in the works of Putman and Dennis [7], or Nicoud and Poinsot [8] that the flame stability depends on an extended Rayleigh criterion that compares the source term of the acoustic energy from the flame with the acoustic fluxes at the boundary surfaces, as shown in Eq. (1). The source term depends on the phase relationship between pressure and heat release oscillations in the combustor which is imposed by the different time delays appearing in the $\mathrm{p}^{\prime}-\mathrm{q}^{\prime}$ coupling. 


$$
\frac{1}{T} \int_{T}\left\{\frac{\gamma-1}{\gamma \bar{p}} \oiiint_{\Omega} p^{\prime} q^{\prime} d V-\oiint_{\Sigma} p^{\prime} u^{\prime} d A\right\} d t>0
$$

In Eq. (1), $\gamma$ is the heat capacity ratio; p', u', q' are, respectively, the pressure, velocity, and heat release rate fluctuations; $\mathrm{T}$ is the instability cycle period; $\bar{p}$ is the average pressure over the system control volume, $\Omega ; \Sigma$ is the system control surface; and, finally, $\mathrm{dV}$ and $\mathrm{dA}$ are, respectively, the volume and surface integration variables.

Consequently, in order to evaluate the coupling risks from CFD, a good prediction of the time delays, and thus of the physics involved, is necessary

In the literature, many research works deal with gas-fueled combustors in premixed or diffusion regimes in order to describe and model the phenomena considered and to determine the p' (or u') - q' relationship [9, 10]. When a liquid fuel is used, the coupling between the acoustic fluctuations and the unsteady heat release involve additional phenomena, such as spray atomization / transport / vaporization / combustion [11-14] and their interaction with turbulence, vorticity, chemistry, and acoustics. Eckstein et al. [11] analyzed the spray generated by an air-blast system subjected to periodic air velocity fluctuations. The authors observed that the droplet size in the spray varied periodically, at the same frequency as the velocity excitation. Moreover, they explained that, for a given liquid flow rate, high air velocities produce a large number of small droplets, whereas low air velocities produce a small amount of large droplets. Thus, when combustion instabilities occur, the periodic velocity fluctuations inside the atomizer create a time-varying droplet size distribution, which is transported further downstream to the flame as a droplet wave. During this convection phase, the small-droplet zones produce a larger amount of fuel vapor than the large-droplet zones. As a result, an equivalence ratio wave appears, which interacts with the flame to produce a periodic heat release oscillation.

In parallel, Giuliani et al. [12] and Gajan et al. [13] studied non-reactive spray behaviors downstream both from a simplified atomizer and from a Dextre-type industrial atomizer under non-reactive conditions. In the first case, droplets were formed from the disintegration of an axisymmetric liquid sheet sheared internally and externally by two co-swirling airflows. In the second case, a prefilming zone was formed before the atomization of the liquid fuel. In these experiments, velocity pulsations were created by a siren placed upstream or downstream from the atomizer. In both cases, droplet density waves transported downstream from the fuel injection location were observed and Giuliani [14] observed a great interaction 
between the droplet concentration fronts and the convection of annular vortices formed in the swirling air jet shear layer.

More recently, Apeloig et al. [15] studied the unsteady interaction of a kerosene spray downstream from a multi-point injector with flame. The injection system comprised two injection zones, a pilot zone with a pressure atomizer at the center and a multipoint zone at the periphery. In this zone, the liquid fuel was injected through a set of individual jets in crossflow. When combustion instabilities occurred, phase-averaged processing of images obtained from planar laser-induced fluorescence (PLIF) on kerosene revealed that the spray pattern issuing from the multipoint zone fluctuates to a large extent, both spatially and in terms of fluorescence intensity, over the instability cycle. In particular, it was observed that, at different phase angles of the instability cycle, three distinct droplet fluxes issued from different zones appear (Fig. 2). Apeloig showed that these unsteady phenomena are at the origin of heat release fluctuations measured in the flame. From the analysis of these image sequences, Apeloig et al. [15] proposed that the droplet flux oscillations are linked to a periodic bending of the individual liquid fuel columns inside the injector, induced by the local air velocity fluctuations. In particular, depending on the instantaneous liquid-to-air momentum flux ratio, each liquid jet may impact the opposite injector wall forming a liquid film, which is atomized further downstream at the injector outlet. Different characteristic times thus appear, due to the diverse convective mechanisms involved in the liquid atomization and transport from its injection point inside of the injector (wall filming or droplet transport) to the combustor. These time scales have to be taken into account in flow simulations used for the flame transfer function determination.

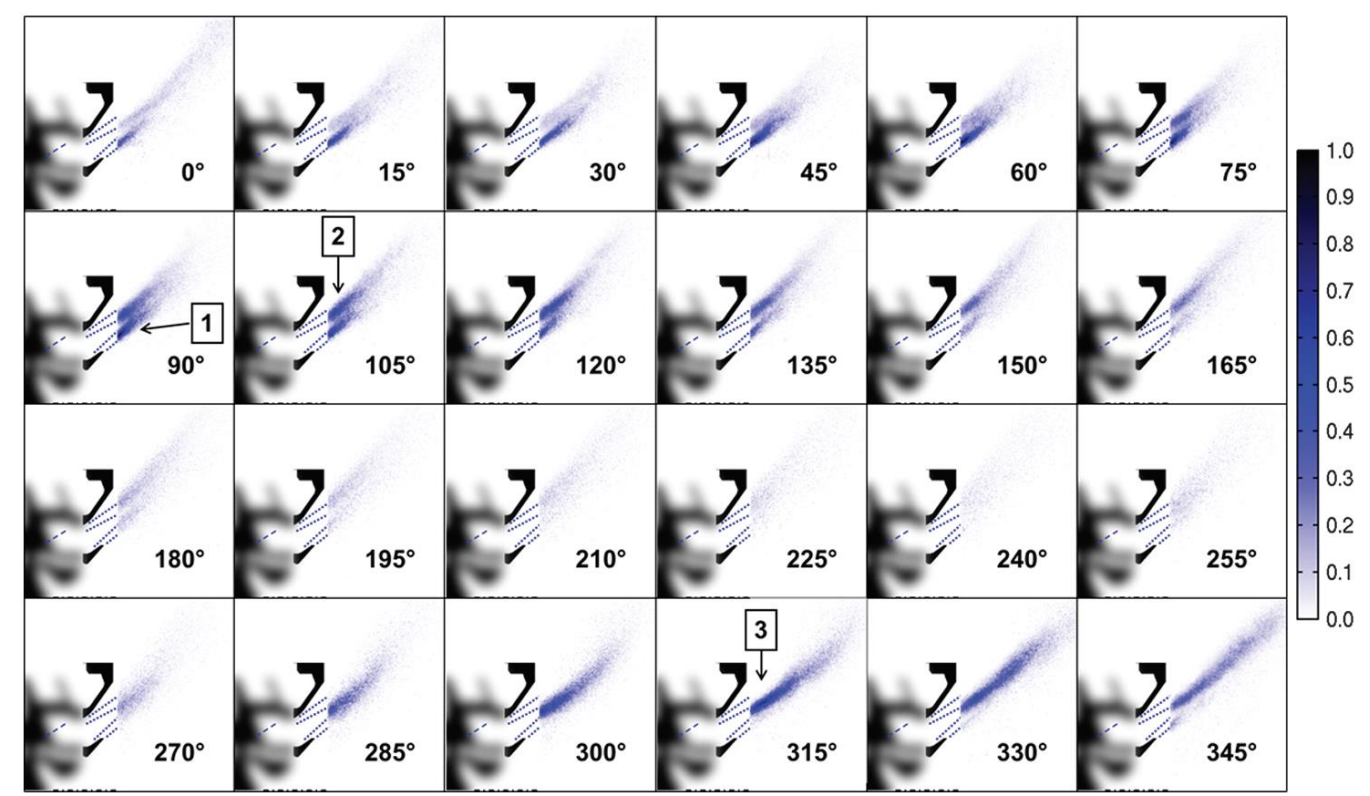


Fig. 2: Temporal sequence of the liquid kerosene spatial distribution during an instability cycle using phaseaveraged analysis on PLIF images (Apeloig et al. [15])

The understanding and simulation of the flow phenomena observed during works on multipoint injection devices are at the origin of the ONERA research project SIGMA ${ }^{1}$ on combustion instabilities appearing in liquid fuel injection systems. The aim of one the tasks of this project is to study the behavior of a liquid jet in a cross airflow subjected to an acoustic excitation, in order to analyze the main phenomena involved and to provide a detailed database for CFD validation. This paper will present results obtained from experiments. A first paragraph is dedicated to the state of the art on the liquid jet in cross flow with and without acoustic excitation. Then, a description of the experimental setup is presented. The result section begins with a description of the data obtained without acoustical excitation which are compared with the literature. Further, the paper focuses on the analysis of the entire two-phase flow behavior observed with the acoustic excitation, in order to quantify its main characteristics.

\section{State of the art concerning jets in a cross flow}

The behavior of a liquid jet in a cross flow (LJICF) under steady conditions was studied in detail by various authors [16-18]. They showed the influence of various parameters on the liquid behavior. Therefore, the atomization regime of the liquid jet column is controlled by the Weber number We, based on the air velocity and the initial jet diameter. For Weber numbers higher than 110, Sallam et al. [16] observed that a shear breakup regime is obtained. It begins by deflection of the liquid jet but with negligible distortion of the jet cross section. Wavelike disturbances appear on the upstream side of the liquid jet, probably as a result of Rayleigh/Taylor instabilities, and grow into ligaments along the periphery of the liquid jet. These ligaments are then subjected to similar hydrodynamic instabilities which cause their breakup and induce the formation of droplets that are ejected away from the liquid column to form a spray. Wu et al. [17] observed that the moment flux ratio $q$ (Eq. 2) may also impact the critical Weber number corresponding to the occurrence of this shear breakup regime. They also distinguished two atomization modes, the first one on the jet surface and the second at the end of the liquid column.

\footnotetext{
${ }^{1}$ Simulation des Instabilités de combustion Générées dans les Moteurs Aéronautiques et spatiaux
} 


$$
q=\frac{\rho_{l} V_{j}^{2}}{\rho_{G} V_{G}^{2}}
$$

Sallam et al. [16] showed that the sizes of ligaments and droplets depend on the atomization regime and are correlated to a non-dimensional number based on a Weber number and on a liquid-to-gas viscosity ratio $\left(\left(\mu_{/} / \mu_{G}\right)(1 / W e)\right)$.

Many authors have observed that the jet trajectory is mainly influenced by the moment flux ratio. Nevertheless a great dispersion among the results was obtained. Wu et al. [17] noticed that other parameters must be taken into account, such as the liquid flow regime at its inlet outlet, which impacts the Rayleigh-Taylor instability growth on the upwind jet column surface and thus the drag forces.

$\mathrm{Wu}$ et al. [17] investigated the influence of different parameters on the axial $\left(y_{b}\right)$ and transverse $\left(z_{b}\right)$ location of the jet column breakup. They observed that the ratio $y_{b} / d$ is nearly independent of the moment flux ratio $\left(y_{b} / d \sim 8.06\right)$, whereas the transverse location varied as 3.07. $q^{0.53}$.

The influence of acoustic forcing on LJICF was studied by Anderson et al. [19], Bunce et al. [20], Song et al. [21] and Sharma and Lee [22]. It depends on the relative location of the liquid jet injection with respect to the acoustic pressure and velocity nodes or antinodes [20], and on the excitation level and the frequency of the air flow excitation [21]. With a liquid jet injected close to a pressure node, Bunce et al. did not observe any oscillation of the jet trajectory. The excitation level defined by $\tau=u_{r m s}^{\prime} / U_{0}$ ( $U_{0}$ represents the bulk velocity) is equal to about $35 \%$ in the work by Anderson et al. [19], whereas values between 5\% and 20\% are reported by Song et al. [21]. Whereas Anderson et al. [19] obtained a huge oscillation of the liquid column linked with an influence on the spatial distribution of the spray particle number density, Song et al. [21] observed very little influence of the excitation on the jet trajectory but a periodic variation of the droplet size distribution. More recently, Sharma and Lee [22] studied the influence of the excitation frequency on the liquid jet response. They observed that the liquid jet and spray oscillations decrease as the frequency increases. To explain this phenomenon, they compared the excitation period with the jet breakup time scale given by Wu et al. [17]:

$$
t_{b}=3.07\left(\frac{d_{j}}{V_{j}}\right) q^{0.53}
$$

If this jet time scale is very small compared to the modulation period, the whole liquid jet encounters a particular phase in the crossflow fluctuation and responds to it before break up. Conversely, if the two timescales are equivalent, the liquid mass will experience varying 
phase of the crossflow oscillations and consequently it will have a more uniform response [22].

\section{Experimental approach}

The experimental setup dimensions and the flow conditions were chosen to reproduce the main phenomena observed by Apeloig et al. [15] within the injection system and described in the introduction. Therefore, the Weber number ensures an equivalent atomization regime while both the momentum flux ratio and the channel height allow the observation of periodic liquid impacts on the upper wall.

\section{SIGMA experimental set-up}

The SIGMA experimental set-up shown in Fig. 3 was designed to obtain an air flow subjected to high acoustic levels. The air flow issued from a high pressure tank was directed into a plenum placed upstream of a pneumatic loudspeaker working in the sonic regime. The mass flow rate is directly linked to the pressure and the temperature measured in the plenum with a K-type thermocouple and a gauge pressure. This system was calibrated beforehand and an accuracy greater than $2 \%$ was obtained. The test model was connected to the pneumatic loudspeaker through a $2 \mathrm{~m}$ long straight pipe having a $50 \times 50 \mathrm{~mm}^{2}$ square cross section. The overall pipe length downstream from the pneumatic loudspeaker was defined, first to permit the flow development up to steady conditions and then to obtain acoustic modes within the frequency range observed in combustion chambers (between $100 \mathrm{HZ}$ to $600 \mathrm{~Hz}$ ). The outlet liquid jet was placed close to a velocity antinode corresponding to high air velocity fluctuations, in order to maximize the influence of the excitation.

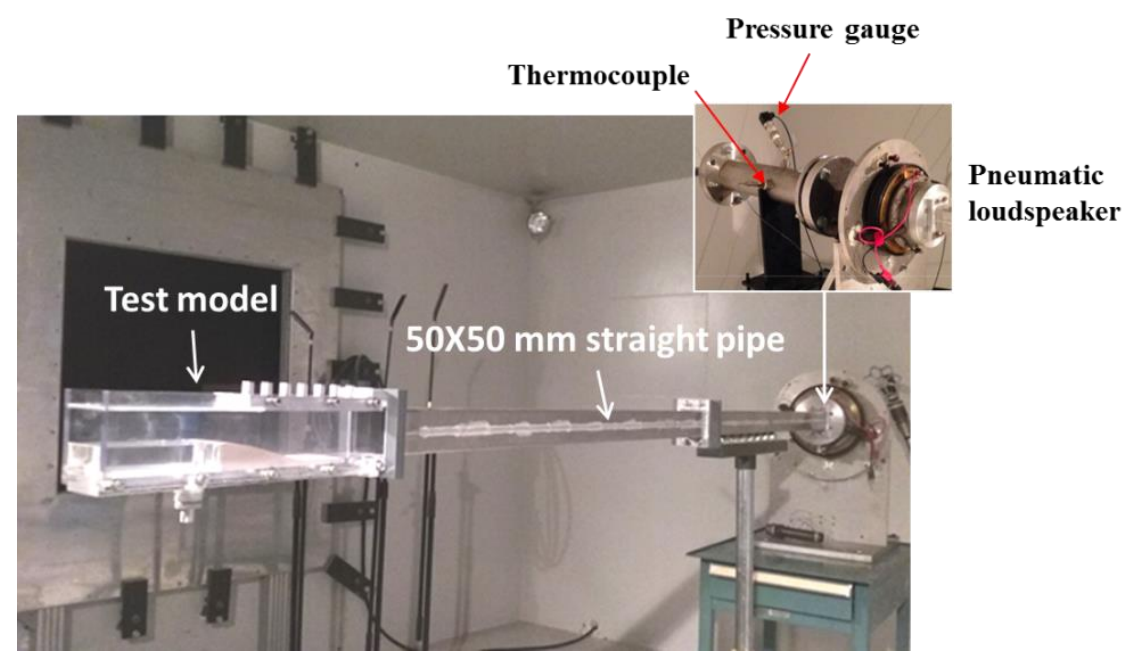

Fig. 3 : Sketch of the SIGMA setup 
The test model is sketched in Fig. 4. It consists of three zones. The inlet section, $100 \mathrm{~mm}$ long, has the same cross section as the upstream duct. It is followed by a convergent section ensuring a channel height reduction with a smooth transition without airflow separation. The third part having a rectangular cross section $\left(20 \times 50 \mathrm{~mm}^{2}\right)$ corresponds to the test section. It is $110 \mathrm{~mm}$ long. At its end, air and liquid flows exit into the room at ambient conditions.

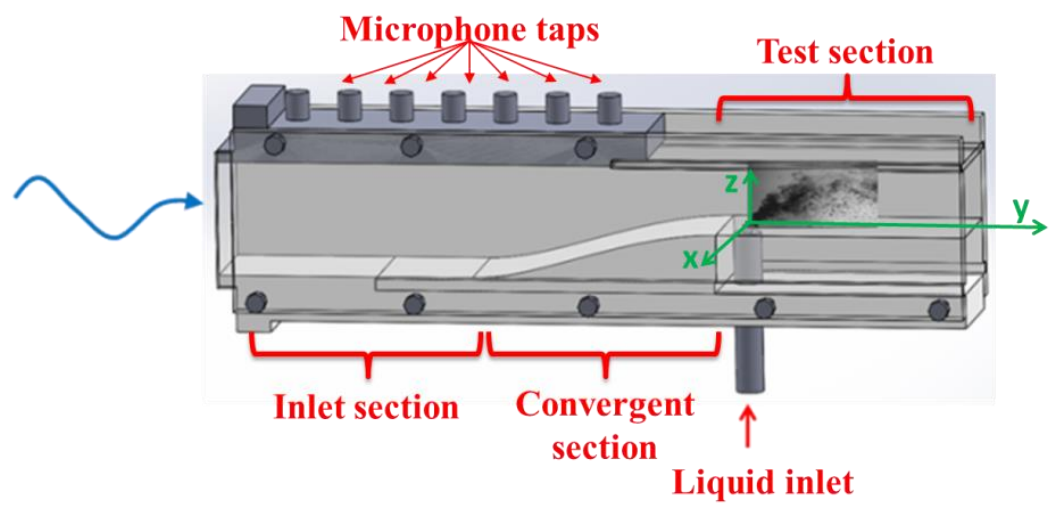

Fig. 4 : SIGMA test model

For visualization and laser diagnostics, the vertical walls of the test section are made with Perpex. A glass window is located on the top of the test section. A set of 7 microphone taps placed as shown in Fig. 4, enables the acoustical characterization of the setup.

A water liquid jet is injected vertically upwards in the symmetry plane of the test model, flush with the floor wall. The origin of the Cartesian coordinate system used in this paper corresponds to the liquid jet exit center. The nozzle is placed $100 \mathrm{~mm}$ upstream of the test section exit and $10 \mathrm{~mm}$ downstream from the convergent exit. The liquid injection system consists of a large tank, a pump, a flowmeter and a nozzle. The liquid flow rate is measured with a Coriolis Micro Mass Flow Sensor and controlled with a pump (Micropump 75211-30). The measured mass flow rate is used to calculate the jet initial velocity $\left(V_{j}\right)$ at the nozzle exit. The nozzle geometry is adapted from [17], in order to guarantee a laminar jet. The nozzle passage has an inlet diameter of $6 \mathrm{~mm}$ followed by a $45^{\circ}$ reduction to reach the specific nozzle exit diameter $(d=2 \mathrm{~mm})$, and a straight section with a length/diameter ratio of 4.

\section{Experimental conditions}

The experimental conditions considered for this study and their respective accuracies are summarized in Table 1. Note that, following the classification given by Sallam et al. [16], the 
primary breakup regime always corresponds to the shear breakup mode (characterized by a large Weber number, We >110) throughout this study.

\begin{tabular}{|l|c|c|}
\hline \multicolumn{1}{|c|}{ Parameters } & Values & Uncertainty [\%] \\
\hline Air flow rate $[\mathrm{g} / \mathrm{s}]$ & 75.7 & 1.8 \\
\hline Air bulk velocity in the inlet section $\mathrm{U}_{0, \text { is }}[\mathrm{m} / \mathrm{s}]$ & 25.2 & 1.8 \\
\hline Air bulk velocity in the test section $\mathrm{U}_{0}[\mathrm{~m} / \mathrm{s}]$ & 63 & 1.8 \\
\hline Test liquid & Water & 0.1 \\
\hline Liquid flow rate $[1 / \mathrm{min}]$ & 1.13 & 0.2 \\
\hline Liquid velocity $\mathrm{V}_{\mathrm{j}}[\mathrm{m} / \mathrm{s}]$ & 6.1 & - \\
\hline Momentum flux ratio $q[-]$ & 7.8 & - \\
\hline Weber Number $W e[-]$ & 136 & - \\
\hline Reynolds number of the air in the test section $R_{\text {air }}[-]$ & 21500 & - \\
\hline Reynolds number of the jet $R e_{j e t}[-]$ & 12200 & - \\
\hline Airflow modulation frequency $[\mathrm{Hz}]$ & 177 & \\
\hline
\end{tabular}

Table 1. Experimental conditions

\section{Experimental methods}

Various techniques were implemented to analyze the flow behavior. In a first approach the acoustic field was identified from sound pressure signals delivered by a microphone at different longitudinal locations on the upper wall of the test model. From the combined use of a 1D model and pressure data processing, the acoustic condition at the channel exit, the longitudinal distribution of the velocity, and the pressure amplitudes were determined.

A Phase Doppler Anemometer (PDA) from Dantec Dynamics was used to characterize both the mean air flow and the velocity oscillations. It comprises an emission module (Flow Master) and a collection module (Fiber PDA). The airflow was previously seeded with particles of DEHS with a diameter smaller than 1 micron.

Two different techniques allow the liquid behavior to be studied. The first uses high-speed visualizations and the second concerns droplet size and velocity measurement with the same Phase Doppler Anemometer (PDA) previously described. During tests, two zones were considered; the first is located close to the liquid jet injection while the second concerns the test section exit. A back lighting method (two Dedocoo lamps) was used to light the flow, and the images were recorded by a high-speed black and white camera (Phantom V341). The 
camera was coupled with a $50 \mathrm{~mm}$ objective lens. The field of view was limited to $1900 \times 500$ pixels, in order to maintain a good spatial resolution. It was operated at different frame rates ranging between 800 and $10000 \mathrm{~Hz}$, in order to capture flow features with different characteristic times. The integration time was kept generally below $50 \mu \mathrm{s}$, in order to freeze the motion of the liquid column and the droplets. Multiple videos were recorded for each test condition. Dedicated software was developed to analyze the liquid behavior.

Given that different experimental techniques were used to analyze the flow behavior induced by the acoustic excitation, a statistical analysis of the experimental data is needed. The phaseaveraged analysis is the most common method to study cyclic phenomena, in particular in combustion experiments. Nonetheless, a periodical reference signal is needed to perform this analysis. Here, the velocity signal at the location of the liquid jet exit is considered. It is deduced, after a calibration, from the excitation signal used to pilot the pneumatic loudspeaker. PDA and LDA post processing were performed on $10^{6}$ particles with a phase angle step of $4^{\circ}$, whereas 38428 instantaneous images were considered with a phase angle step of $10^{\circ}$ and a bandwidth of $4^{\circ}$. Thus, from the phase-averaged processing, each signal $f(\boldsymbol{X}, t)$ is decomposed through 3 quantities $\bar{f}(\boldsymbol{X}), \tilde{f}(\boldsymbol{X}, t), f^{\prime}(\boldsymbol{X}, t)$ (Hussain and Reynolds [23]), which correspond to the mean, the periodic and the random components of the signal, respectively. The amplitudes of the time-dependent components of the signal are then described through the two following quantities $\tilde{f}_{r m s}$ and $f_{r m s}^{\prime}$. The first quantify the amplitude of the periodic oscillation linked to the acoustic forcing, while the second yields information regarding the random component of the signal.

\section{Results}

In this section, the acoustic framework of the set-up is first presented with information concerning the air flow characteristics. Then, the behavior of the liquid is analyzed from the visualization and PDA results.

\section{Analysis of the aeroacoustics field}

The air flow characterization was performed for the air flow condition presented in Table 1 with and without acoustic modulation. The modulation frequency was determined from a 1D analysis of the acoustic pressure and velocity distribution of the main longitudinal mode of the experimental set-up (plane mode). 
The air flow was characterized without liquid injection in different planes from the upstream side of the convergent to the liquid jet injection section. Vertical profiles obtained in the symmetry plane of the channel $(x=0 \mathrm{~mm}), 56 \mathrm{~mm}$ upstream of the convergent inlet and in the liquid jet injection plane are plotted in Fig. 5. Upstream of the convergent, the mean velocity and random intensity distributions were compared and were found to correspond to Brundrett and Baines results [24] obtained for a fully developed turbulent flow in a square channel. Therefore, the mean velocity profile may be described by a power law. In parallel, the $u_{r m s}^{\prime}$ intensity linked to the flow turbulence is minimum on the channel axis $\left(u_{r m s}^{\prime} / U_{o, i s}\right.$ around $4 \%)$ and maximum near the walls (10\%). The intensity of the periodic component is uniform along the $\mathrm{z}$ axis $\left(\tilde{u}_{r m s} / U_{o, i s}=3.4 \%\right)$ corresponding to a plane wave. At the jet outlet location, a flatter mean velocity profile is obtained. The random fluctuations are lower compared to the upstream section, except near the bottom wall. Conversely, periodic fluctuations linked to the acoustic forcing are amplified. On the central part of the channel, the spatial distribution remains uniform. Near the lower wall, the increase observed may be due to the behavior of the boundary layer in this zone.
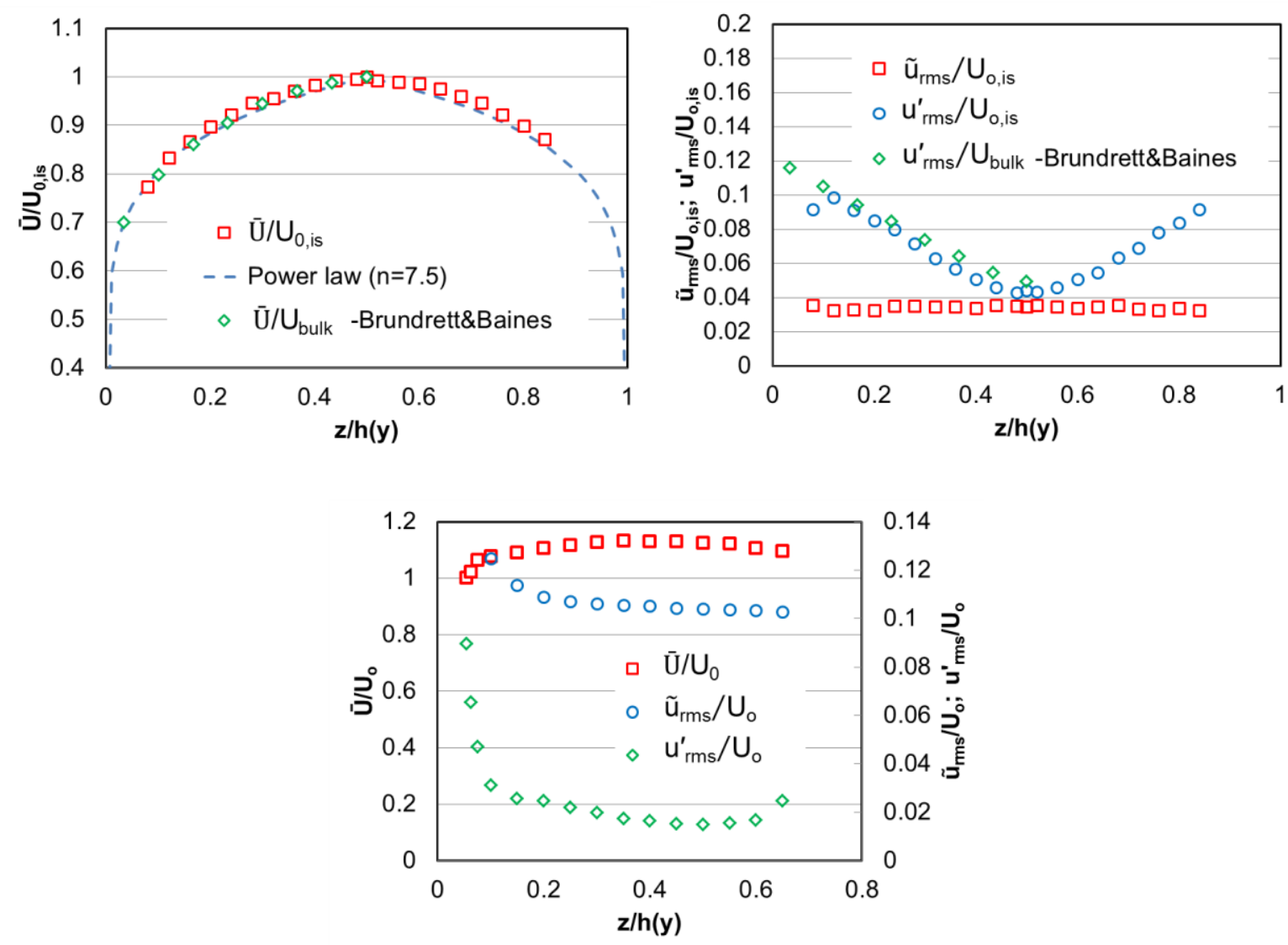

Fig. 5. Velocity profiles measured upstream of the convergent inlet and in the liquid jet injection plane. Top images: upstream of the convergent section; Bottom image: liquid jet injection plane 
The 1D model allows different incident and reflected wave lengths due to air mean flow [25] to be taken into account. The aim was, firstly, to generate high pressure stationary waves at a duct resonance frequency and, secondly, to maximize air velocity fluctuations close to the liquid jet outlet.

From microphone and LDA measurements, the longitudinal shape of the pressure and velocity waves are measured and compared to the 1D model, with a hypothesis of plane waves (Fig. 6). It can be observed that the 1D model yields results that are in very good accordance with the experimental data upstream of the test section, but they are slightly different further downstream. One can notice that the flow is sufficiently fast to increase the pressure amplitude at the nodes. The procedure used to measure the reflection coefficient is based on the approach proposed by Åbom and Bodén [26] for plane incident waves in the direction of the flow. According to this approach the acoustic pressure is expressed as:

$$
p^{\prime}\left(y^{\prime}, t\right)=\left(A e^{-j k^{+} y^{\prime}}+B e^{j k^{-} y^{\prime}}\right) e^{j \omega t} \text { for } y^{\prime}=y-0.1
$$

with $k^{+}=\frac{\omega}{c(1+M)}, k^{-}=\frac{\omega}{c(1-M)}$, and $M=\frac{u}{c}$

The outlet reflection coefficient, expressed as $R=B / A$, is close to 1.3 at the excitation frequency. This quantity is possibly higher than 1 because the reflected wavelength is lower than the incident one (cf. $k^{+}$and $k^{-}$). It can be compared to its theoretical value of 1.47 corresponding to a complete reflection of the acoustic power in flow $(\mathrm{P}=0)$ for which $R_{\max }=\frac{1+M}{1+M}$ [25] (cf. air bulk velocity $\mathrm{U}_{0}=63 \mathrm{~m} / \mathrm{s}$ and ambient conditions). This means that a part of the acoustic energy escapes from the channel. The amplitude of the pressure fluctuations up to $1900 \mathrm{~Pa}(160 \mathrm{~dB})$ is measured upstream of the convergent section while the amplitude of r.m.s. velocity fluctuation is around $6.5 \mathrm{~m} / \mathrm{s}$ at the liquid jet exit location corresponding to a modulation level of $10 \%$ with respect to the air bulk velocity in the jet exit plane $\mathrm{U}_{0}$. 


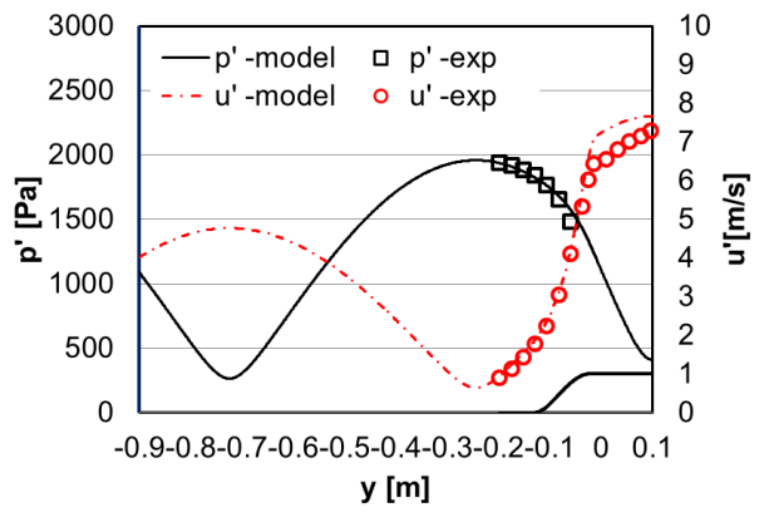

Fig. 6. Longitudinal distribution of the acoustic pressure and velocity in the test model.

\section{Characteristics of a liquid jet in a steady flow}

Fig. 7 illustrates the main features of the liquid flow behavior observed for a crossflow without acoustic excitation. The jet bends towards the leeward direction and impacts the upper wall of the channel, which leads to the formation of a liquid film, as shown in Fig. 7(a). This liquid film spreads along the wall and is finally atomized by the air flow at the channel exit, as shown on Fig. 7(c).

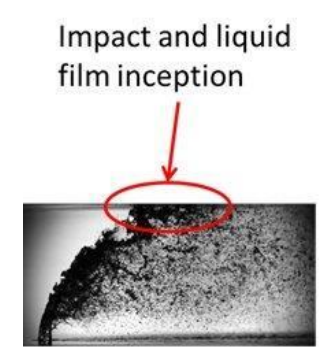

(a)

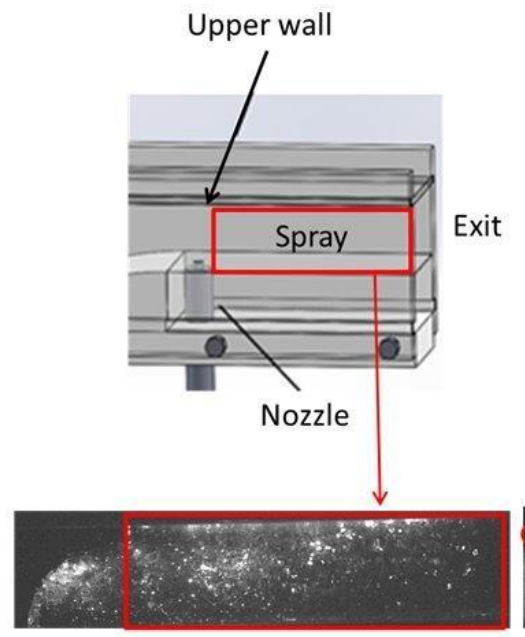

(b)

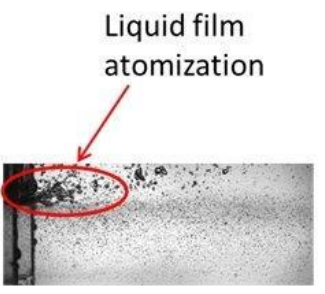

(c)

Fig. 7. Flow features: (a) Liquid jet injection into the crossflow at the nozzle location and impact on the upper wall resulting in the creation of a liquid film; (b) spray induced by the liquid jet atomization; (c) atomization of the liquid film at the channel exit.

Shadowgraph visualization allows the liquid jet behavior and its breakup process to be characterized. The data processing is focused on two main features usually studied in this configuration: the liquid column trajectory and the column breakup point location. 
The trajectory of the liquid column was extracted from averaged shadowgraph images and is defined as the upper surface of the liquid column. Fig. 8 shows the typical column trajectory modifications induced by momentum flux ratio $q$ variations. As shown in Fig. 8 (a) and (b), waves appear along the surface of the liquid column and aerodynamic drag forces accelerate the windward surface of the liquid jet, and amplify the surface waves and the surface shearing. This leads to both column and surface breakup processes generating different liquid structures like ligaments and droplets. A large part of these structures directly impacts the upper wall and the remaining part is carried away by the airflow and undergoes a subsequent atomization into smaller droplets. In Fig. 8 (c), the spray induced by the jet atomization process shows smaller droplets and fewer ligaments compared to Fig. 8 (a) and (b). This is due to the confined configuration. In this case, the column breakup does not occur, because the liquid column directly impacts the upper wall before the column break point.
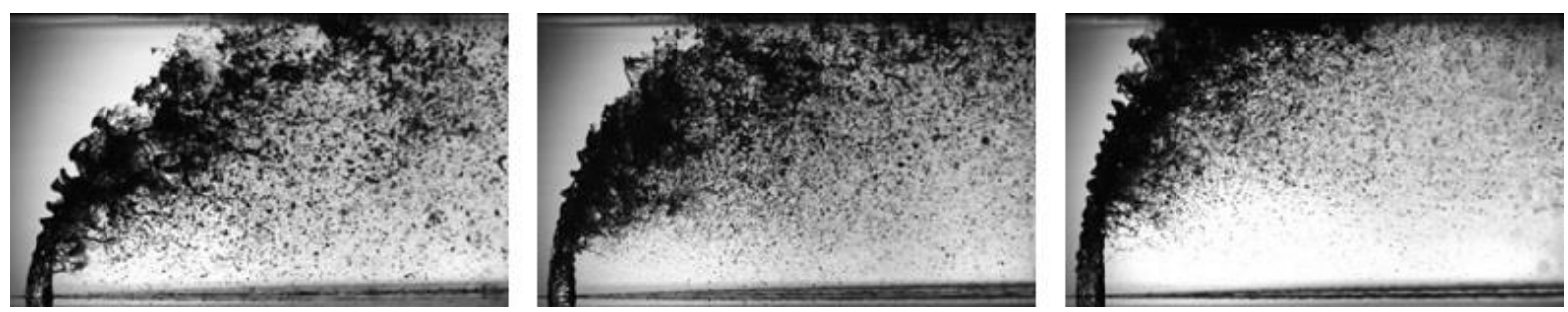

Fig. 8. Injection of the liquid jet into the crossflow at various momentum ratios $($ We=136). Left: $q=7.8$; Middle: $q=11.8$; Right: $q=17.5$.

Fig. 9 shows the liquid column trajectory for various momentum flux ratios $q$ and for a fixed Weber number $W e=136$. The results show a higher penetration of the liquid column as the parameter $q$ increases, which is in accordance with the results obtained by Wu et al. [17]. The trajectories from the present study are in agreement with $\mathrm{Wu}$ et al. [17] for maximum three jet diameters, but further downstream a smaller liquid jet penetration is observed. These differences can be explained by the confinement effect, the difference between measurement techniques and the accuracy of the image processing technique used to define the liquid jet boundaries, which depends on the image contrast and the threshold taken into account. 


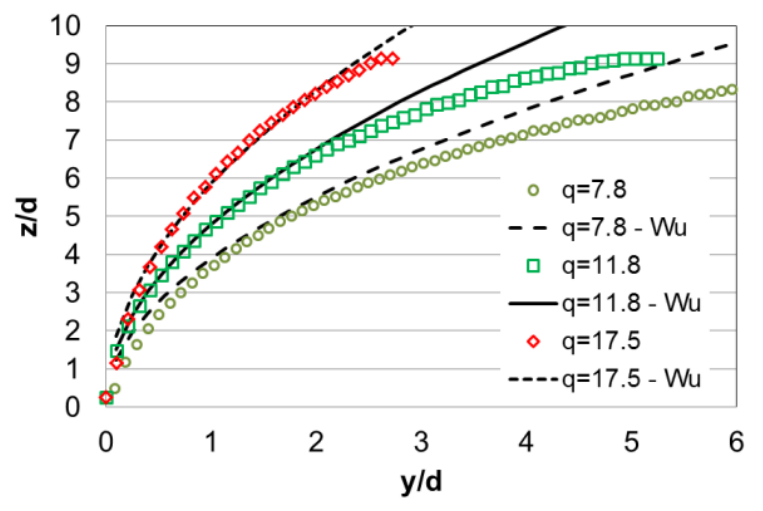

Fig. 9. Liquid column trajectories for a steady crossflow and for various momentum ratios $(W e=136)$.

Another liquid jet characteristic obtained by image processing was the longitudinal location of the column fracture point $y_{b} / d$. The locations obtained for different momentum flux ratios were fond to be in the range of values reported by Wu et al. [17] $\left(y_{b} / d \sim 7.8\right.$ versus $y_{b} / d \sim 8$ in the reference paper).

\section{Characteristics of the liquid jet in a modulated airflow}

This section focuses on the characteristics of the liquid jet injected in a modulated air flow. On video recordings, a periodic swinging phenomenon of the liquid column is observed, linked to a cyclic droplet atomization from the jet column. This observation is valid only for a modulated crossflow. Unlike the steady crossflow configuration, the impact of the liquid jet on the upper wall is cyclical and most of the ligaments and droplets induced by the column breakup process are directly carried away by the crossflow without impacting the upper wall.

The local jet behavior under crossflow modulation is characterized by spectral analysis of the instantaneous grey level signals obtained at different locations from Mie-scattering images. The results showed that, whatever the phenomena observed or the channel location, the liquid phase mainly responds to the acoustic forcing.
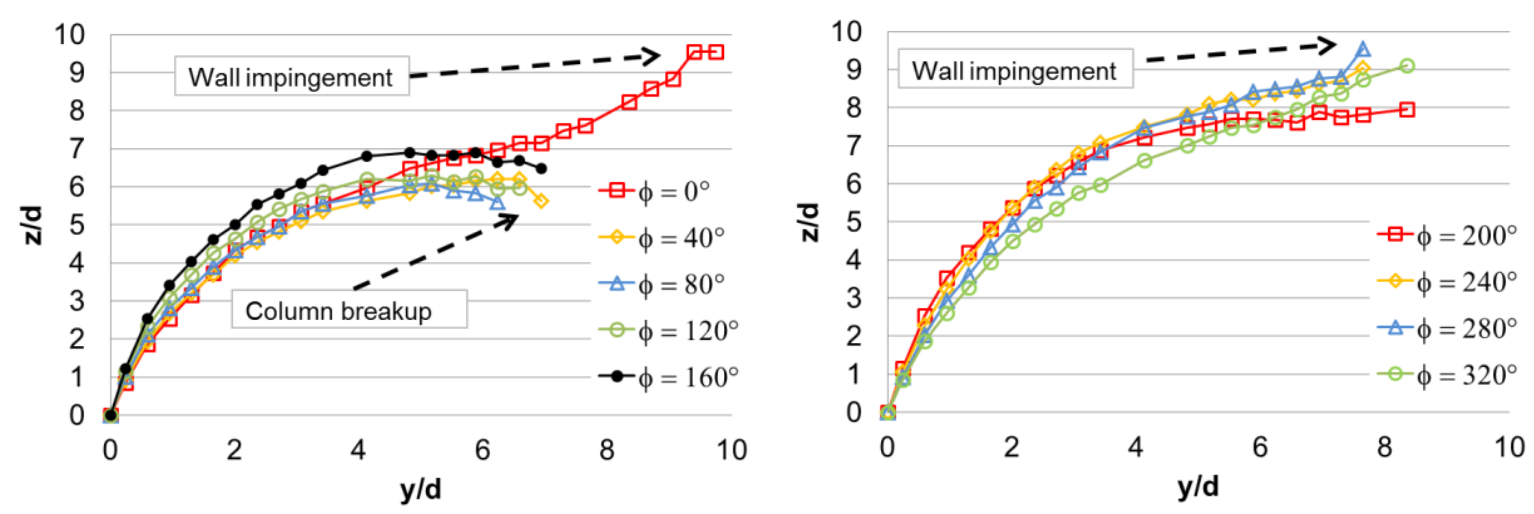
Fig. 10. Liquid jet trajectory evolution with respect to the acoustic disturbance phase measured at $\mathrm{y} / \mathrm{d}=0(q=7.8$, We=136).

The movement of the phase-averaged liquid jet trajectory during the air velocity cycle is illustrated in Fig. 10. During the air velocity acceleration $\left(0^{\circ}<\phi<180^{\circ}\right)$, the liquid column bends towards the floor, while it straightens back during the deceleration phase. The two important points to observe for the purposes of combustion instability are the phase delays corresponding to the breakup of the liquid column and the liquid deposition on the upper wall. Concerning the first point, the breakup happens for phase angles in the range of $20^{\circ}<\phi<30^{\circ}$, corresponding to the beginning of the air deceleration $\left(\partial^{2} u / \partial t^{2}<0\right)$. The liquid deposition linked to the liquid column impact on the upper wall is observed approximately between $\phi=200^{\circ}$ and $\phi=240^{\circ}$.

Fig. 11 illustrates the evolution the liquid jet breakup location with the phase of the acoustic disturbance. The results confirm that the column fracture occurs for the phases between $20^{\circ}$ and $30^{\circ}$. The rupture location is around $7 d$ downstream from the jet exit, and $6 d$ above the floor. Comparisons between liquid jet behaviors obtained with or without excitation suggests that the acoustic forcing does not modify the location of the liquid column breakup, but imposes a cyclic droplet ejection into the air flow with a given phase delay with respect to the velocity fluctuations.

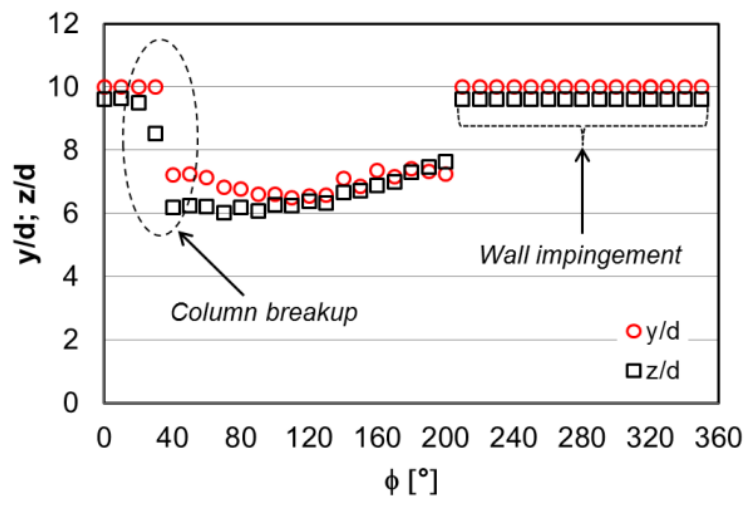

Fig. 11. Evolution of the liquid column breakup position with respect to the acoustic disturbance phase measured at $\mathrm{y} / \mathrm{d}=0(q=7.8, W e=136)$ 


\section{Characteristics of the droplets in a modulated flow}

The PDA technique was then applied to study in more detail the behavior of droplets issued both from the liquid jet atomization and the liquid film separation at the outlet of the test model. The measurement grid used is shown in Fig. 12.

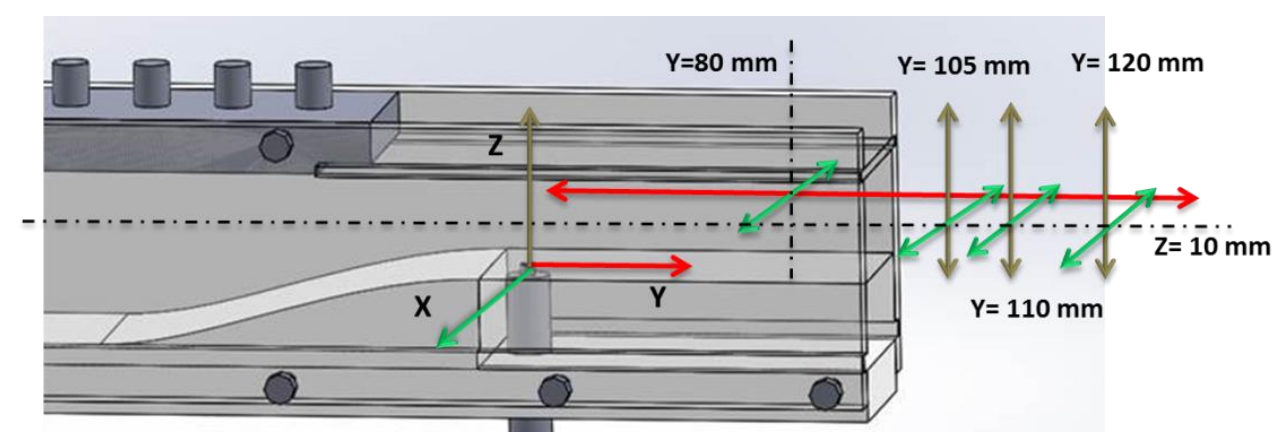

Fig. 12. PDA measurement grid.

The spatial distribution of the mean longitudinal component of the velocity normalized by the air bulk velocity in the test channel $\mathrm{U}_{0}$ is shown in Fig. 13. It can be observed that the droplet velocity increases continuously downstream in the flow. It reaches a maximum value just after the channel exit, and then decreases further downstream due to the gas expansion. Nevertheless, due to their large size, the droplet velocity does not reach the air velocity.

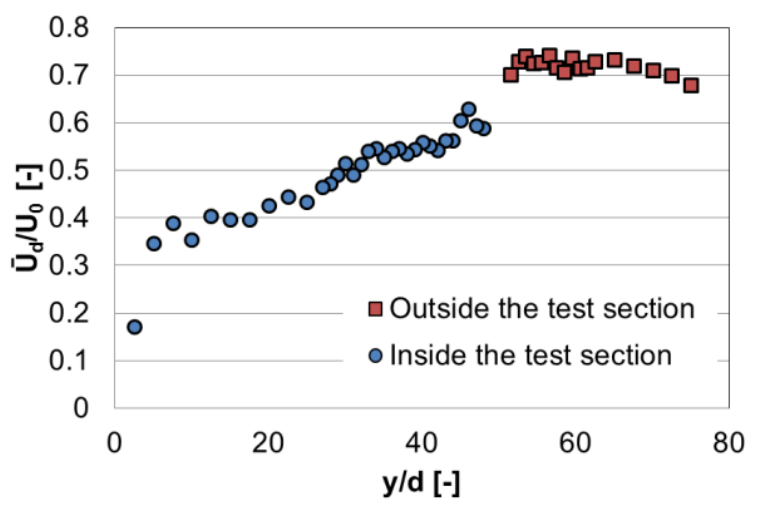

Fig. 13. Evolution of the mean longitudinal velocity of droplets in the duct symmetry plane and for $\mathrm{z} / \mathrm{d}=7.5$.

The phase-averaged droplet velocity signals obtained inside the test channel at different locations downstream from the liquid jet injection point are plotted in Fig. 14. They are compared to air velocity signals obtained at equivalent locations without liquid injection. While the phase angle on the air velocity changes slowly with the distance from the jet exit, an important phase shift is observed on the droplet velocity signal. This phenomenon is induced by the inertia of the droplets. On the right side figure, the modulation of the particle number rate is plotted. This parameter computed from the PDA measurements corresponds to 
the number of particles detected at a given phase angle divided by the average number of particles detected within the whole cycle (overall number of droplets detected divided by the number of phase steps considered). This parameter is representative of a droplet concentration. This graph reveals the formation, close to the liquid jet injection location, of a particle concentration wave that propagates toward the channel exit.
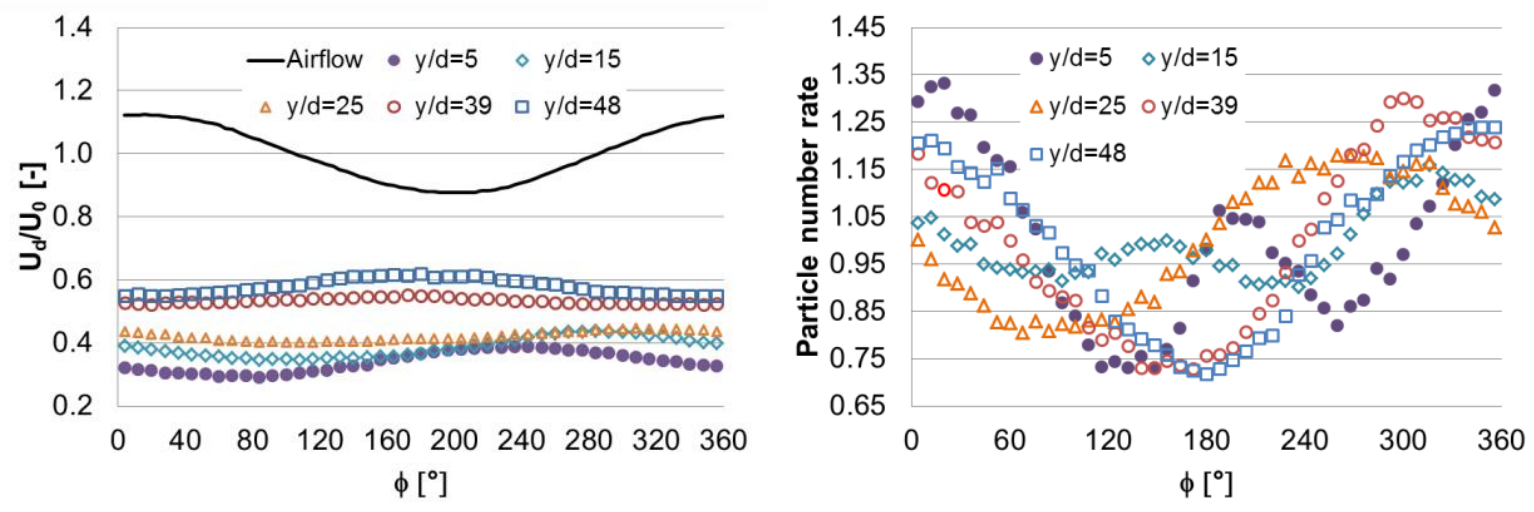

Fig. 14. Cyclic behavior of the spray downstream from the liquid jet exit $(\mathrm{z} / \mathrm{d}=7.5)$. Left: air and droplet velocities; Right: particles number rate

The spray behavior after the channel exit is shown in Fig. 15 and Fig. 16. Some characteristic flow features are highlighted in Fig. 15. Small droplets travelling at a high velocity are observed in the whole channel area, while a liquid film resulting from the liquid jet impact on the upper wall is atomized through the shearing effect imposed by the inner air flow. Concerning this last phenomenon, the liquid film detaches from the wall forming a liquid sheet. A small amount of the liquid is also entrained on the vertical wall. Two different phenomena are thus observed. The liquid entrained on the vertical wall accumulates and forms a liquid bulb that empties periodically at a low frequency around $5 \mathrm{~Hz}$. This phenomenon exists without and with acoustic modulation. The location of the liquid sheet follows the bulb movement. During the liquid accumulation period, it moves above the level of the top side wall and does not interact with the air flow jet. When the reservoir empties, the liquid sheet moves downwards and periodically flaps and interacts with the high velocity air jet issued from the channel. At this moment, large liquid atomization appears, at the excitation frequency, creating dense spray zones that are transported further downstream. The averaged spray characteristics are plotted in Fig. 16. It can be shown that the velocity profile is symmetrical with respect to the channel axis, with a minimum on the centerline and maxima close to the walls. The volume flux gives details concerning the liquid distribution. It can be shown that, in the vertical direction, the maximum liquid flux is obtained in the lower 
part of the channel, while on the horizontal plane it is concentrated close to the centerline. The mean droplet size $\mathrm{D}_{32}$ is around $80 \mu \mathrm{m}$ across the channel section.

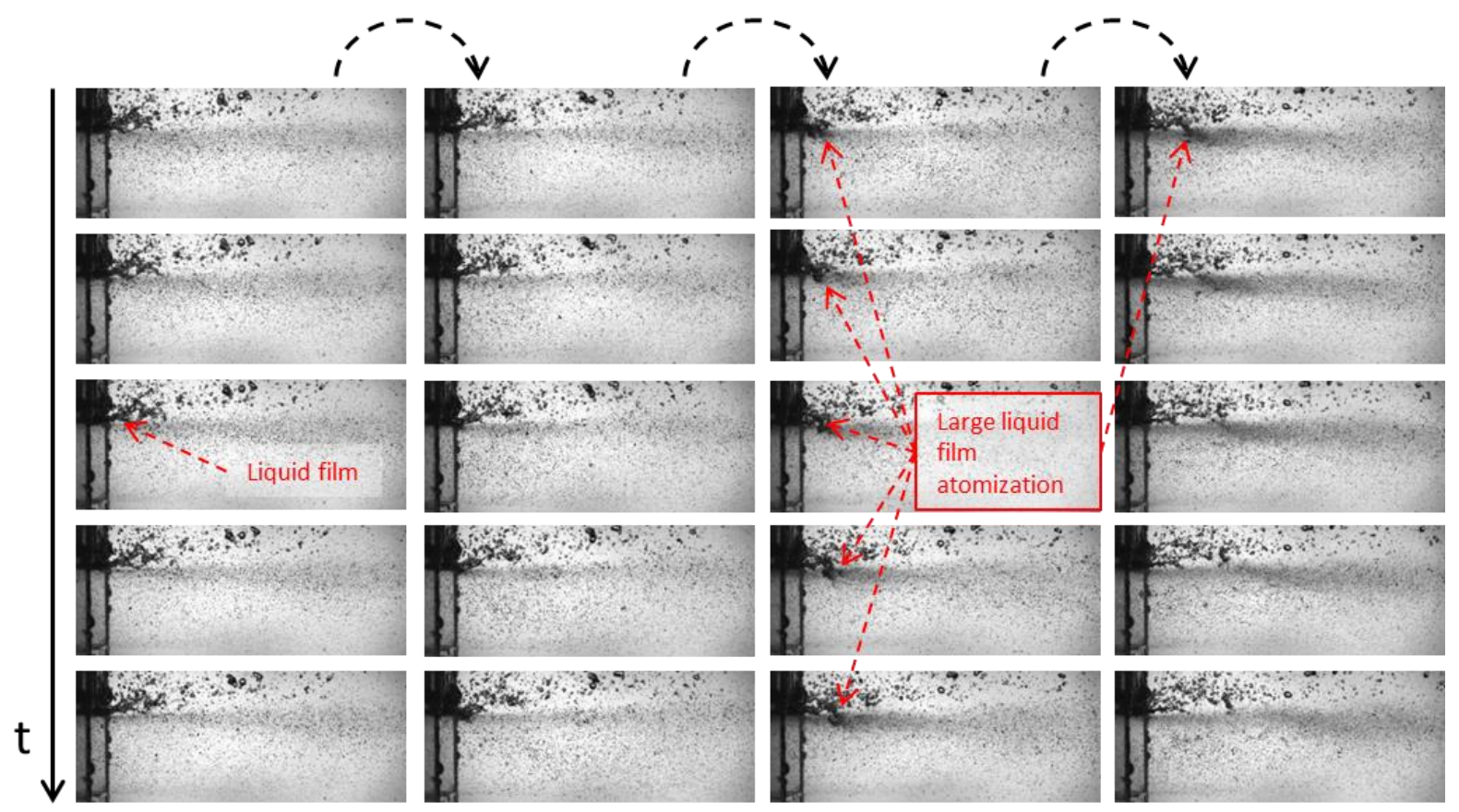

Fig. 15. Visualization of the two phase flow behavior downstream of the channel exit $(W e=192.86, q=7.6$, $\left.\Delta \phi=19^{\circ}\right)$
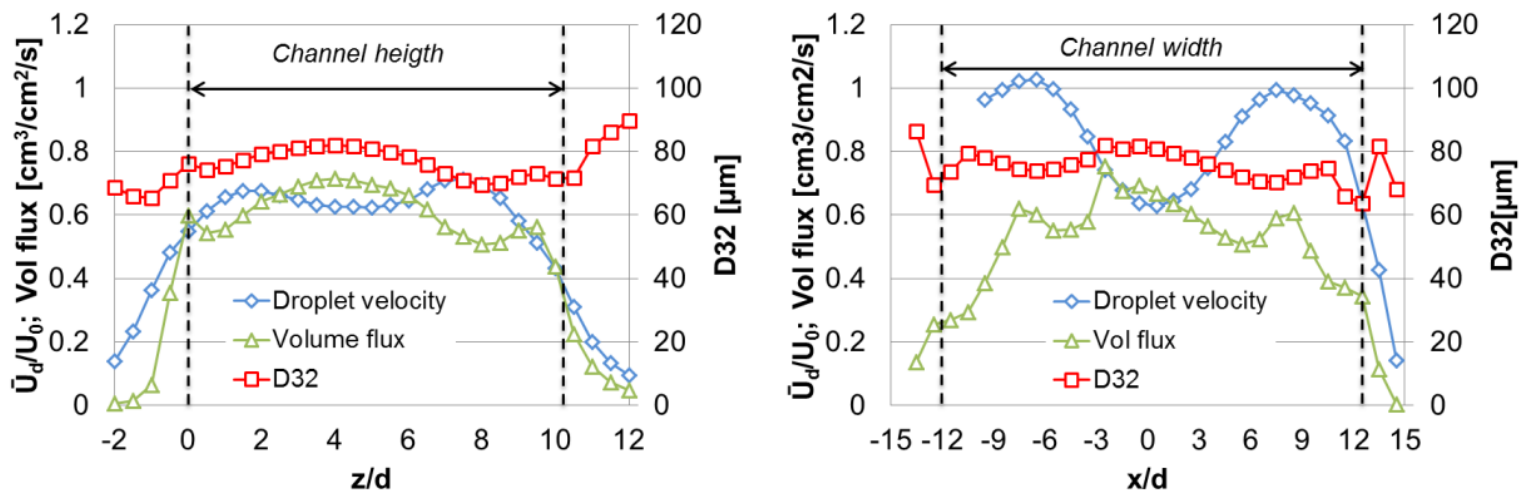

Fig. 16. Droplet velocity, volume flux and droplet size profiles measured at the model outlet (y/d=60). Left: vertical profiles in the duct symmetry plane; Right: horizontal profiles in the duct symmetry plane

Cyclic phenomena observed downstream from the channel exit are analyzed in Fig. 17 in a plane situated at $\mathrm{y} / \mathrm{d}=60$. Three locations are considered, $\mathrm{z} / \mathrm{d}=-0.5$ just under the floor level, $\mathrm{z} / \mathrm{d}=5$ at the height of the channel centerline and $\mathrm{z} / \mathrm{d}=10$ at the height of the top wall. A cyclic arrival of the droplets is obtained with different phase shift linked to the different physical processes involved. An equivalent liquid behavior was observed by Apeloig et al. [15] with a multipoint injector. Different phase delays were also observed on the droplet 
velocity and size signals. On the flow centerline, the minimum velocity corresponds to a large droplet size and low droplet concentration. On the top side, a constant droplet velocity is measured with a cyclic modulation of the droplet size and concentration. When compared with the air velocity signal measured close to this point (Fig. 14), it can be noticed that the higher production of droplets corresponds to high air velocity, and inversely, a low production is obtained when low air velocity occurs. Furthermore, when comparing the droplet number rate and droplet size signals, an out-of-phase behavior is obtained, suggesting that, due to the periodic flapping of the liquid sheet issued from the film, a large amount of small droplets are formed for phase angles around $45^{\circ}$, whereas a small amount of large droplets are formed at phase angles around $270^{\circ}$. The interpretation of the signals obtained along the jet centerline is more complex. It results from the direct atomization of the liquid jet and the droplet transport toward the channel exit. The influence of the droplet size must be considered.
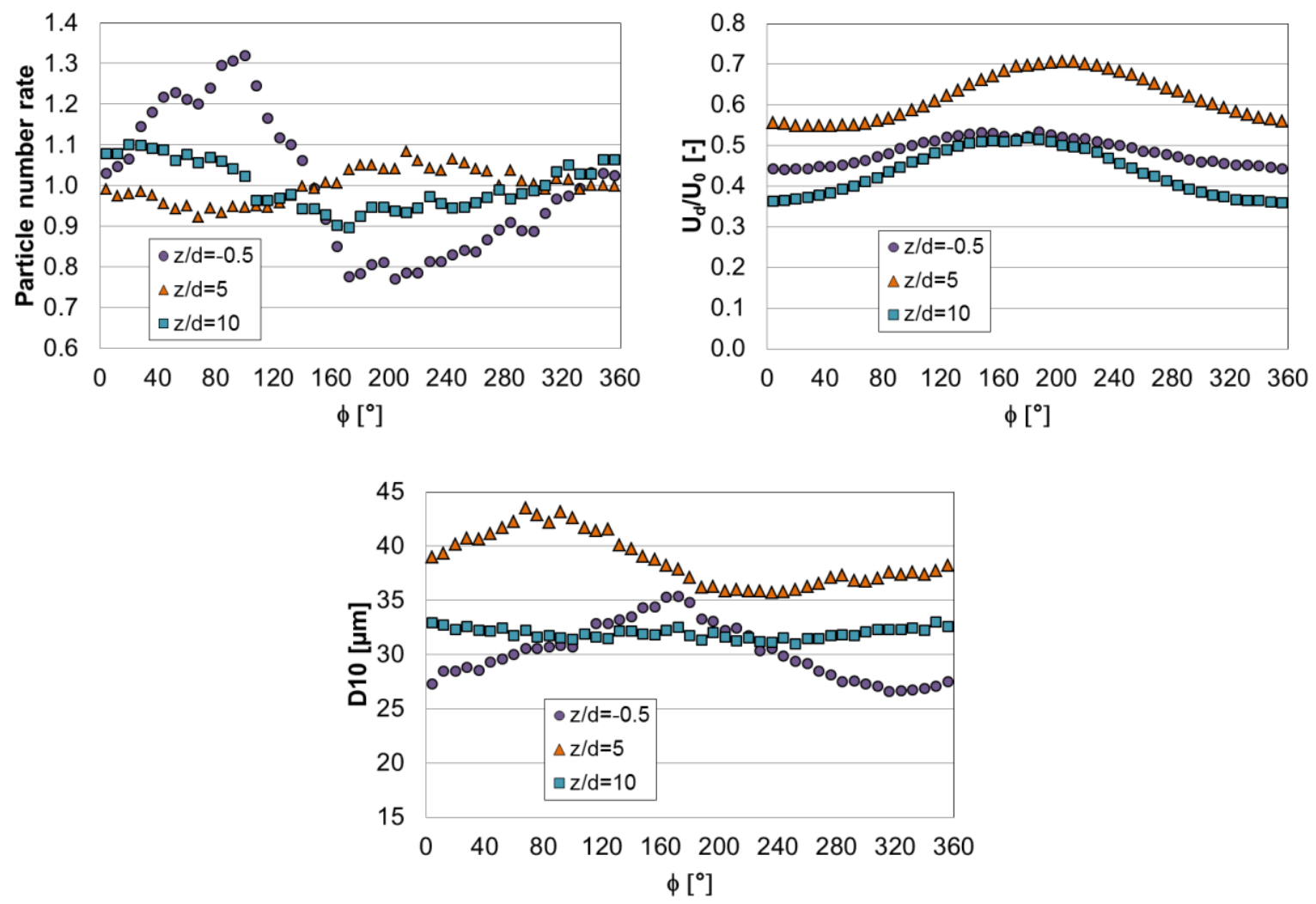

Fig. 17. Cyclic spray behavior at the model outlet $(y / d=60)$ 


\section{Conclusions.}

The experiments presented in this paper reproduce and study in detail fundamental phenomena encountered in multipoint injection devices subjected to high acoustic fluctuations resulting from thermoacoustic instabilities. Different experimental approaches were used to analyze and quantify the main phenomena involved. In a first approach, highspeed video recording processing allows the periodic movement and breakup of the liquid column and the cyclic impact of the liquid jet on the upper wall of the channel to be characterized. The respective time shifts between these different phenomena and the imposed air velocity modulation were measured thereby. Phase-averaging analysis of PDA results reveals harmonic oscillations of the main characteristics of the spray issued from the liquid jet breakup and from direct atomization of the liquid column. The associated convective transport of the droplets by the air flow imposes new phase delays. Downstream from the channel exit, the liquid film resulting from the liquid jet impact induced a new cyclic atomization process characterized by its own phase delay. Thus, this study emphasizes the main periodic phenomena involved and gives detailed inputs for unsteady CFD validations, in particular the time delays associated with each individual behavior. This experimental database will then be used to validate two-phase flow simulation presented in Zuzio et al. [27].

\section{References}

[1] Higgins B. (1802). On the Sound Produced by a Current of Hydrogen Gas Passing Through a Tube. Journal of Natural Philosophy, Chemistry and the Arts, 1, 129-131.

[2] Rijke P. (1859). Notice of a New Method of Causing a Vibration of the Air Contained in a Tube Open at Both Ends, Philosophical Magazine: Series 4, 17(116), 419-422.

[3] Rayleigh J. (1945). The Theory of Sound. New York: Dover.

[4] Harrje D.J, Readon F.H. (1972). Liquid propellant rocket instability. NASA SP 194.

[5] Gajan P., Simon F., Orain M., Bodoc V., Investigation and Modeling of Combustion instabilities in Aero Engines, Aerospace lab Journal, (2016), Issue 11. AL11-9, ONERA

[6] Candel, S., Durox, D., Schuller, T., Palies, P., Bourgouin, J.-F., and Moeck, J. P., Progress and Challenges in Swirling Flame Dynamics, Comptes Rendus Mécanique (2012), 340(11-12), 758-768. doi:10.1016/j.crme.2012.10.024

[7] Putnam A., Dennis W. (1956). A survey of organ-pipe oscillations in combustion systems. J. of Acoust. Soc. of America, 28(2), 246-259.

[8] Nicoud F., Poinsot T. (2005). Thermoacoustic instabilities: Should the Rayleigh criterion be extended to include entropy changes? Combustion and Flame, 142(1-2), 153-159.

[9] Strahle W. (1971). On combustion generated noise. Journal of Fluid Mechanics, 49(2), 399-414. 
[10] Ducruix S., Schuller T., Durox D., Candel S. (2003). Combustion dynamics and instabilities: Elementary coupling and driving mechanisms. Journal of Propulsion and Power, 19(5), 722-734.

[11] Eckstein, J.; Freitag, E.; Hirsch, C.; Sattelmayer, T.: Experimental study on the role of entropy waves in low-frequency oscillations in a RQL combustor. Journal of Engineering for Gas Turbines and Power 128 (2), 2006, 264-270

[12] Giuliani F., Gajan P., Diers O., Ledoux M. (2002). Influence of pulsed entries on a spray generated by an airblast injection device: An experimental analysis on combustion instability processes in aeroengines.

Proceeding of the Combustion Institute, 29(1), 91-98.

[13] Gajan P., Strzelecki A., Platet B., Lecourt R., Giuliani F. (2007). Investigation of Spray Behavior Downstream of an Aeroengine Injector with Acoustic Excitation. Journal of Propulsion and Power, 23(2), 390397.

[14] Giuliani F. (2002). Analysis on the behaviour of an aeroengine air-blast injection. Toulouse: PhD Thesis, ISAE.

[15] Apeloig J.M., d'Herbigny H.X., Simon F., Gajan P., Orain M., Roux S. (2015). Liquid-Fuel Behavior in an Aeronautical Injector Submitted to Thermoacoustic Instabilities. Journal of Propulsion and Power, 31(1), 309-319.

[16] Sallam K.A., Aalburg, C. Faeth GM (2004): Breakup of round nonturbulent liquid jets in gaseous crossflow. AIAA journal, 13(1):64-73

[17] Wu P. K., Kirkendall K. A., Fuller R. P. (1997): Breakup processes of liquid jets in subsonic crossflows, Journal of Propulsion and Power, 31(1), 309-319.

[18] Mazallon, J., Dai, Z., and Faeth, G. M. (1999), Primary Breakup of Nonturbulent Round Liquid Jets in Gas Crossflows, Atomization and Sprays, 9 (3), 291-311

[19] Anderson T.J. Proscia W., Cohen J.M. (2001). Modulation of a liquid-fuel jet in an unsteady crossflow. ASME Turbo expo 2001. New Orleans, Louisiana: ASME. Paper N²001-GT-0048.

[20] Bunce K., Lee J.G., Santavicca D.A. (2006), Characterisation of liquid jets-in_crossflow under high temperature, high velocity non-oscillating and oscillating flow conditions, AIAA paper 2006-1225, $44^{\text {th }}$ AIAA Aerospace Sciences Meeting and Exhibit, Reno.

[21] Song J., Ramasubramanian C., Lee J.G. (2015). Characterization of spray formed by liquid jet injected into oscillating air crossflow. ASME Turbo Expo. Montréal, Canada: ASME. Paper N GT2015-43726

[22] Sharma C., Lee J.G. (2018). Dynamics of near-field and far-field spray formed by liquid jet in oscillating crossflow. Atomization and Sprays. 28(1), 1-21.

[23] Hussain A., Reynolds W. (1970) The mechanics of an organized wave in turbulent shear flow. Journal of Fluid Mechanics. 41, part 2, 241-258.

[24] Brundrett E. and Baines W.D. (1964): The production and diffusion of vorticity in duct flow, Journal of Fluid Mechanics, 19(3), 375-394

[25] Munjal, M.L. (1987). Acoustics of ducts and mufflers with application to exhaust and ventilation system design. A Wiley Interscience Publication.

[26] Åbom M., Bodén H. (1988): Error analysis of two-microphone measurements in ducts with flow. Journal of the Acoustical Society of America 83, 2429-2438.

[27] Zuzio D., Thuillet S., Senoner J.M., Laurent C., Rouzaud O., Gajan P. (2017). Multi-solver LES simulation of the atomization of a cross-flow liquid jet in a channel, Colloque INCA, SAFRAN-Tech. 
V. Bodoc, A. Desclaux, P. Gajan, F. Simon, G. Illac

2, avenue Edouard Belin, 31055 Toulouse

Tel.: +33-562252855

Fax: +33-562252583

E-mail: virginel.bodoc@onera.fr 